\title{
Acute and Repeated Toxicological Study of Anti-Inflammatory Herbal Formula, Yeosinsan, in Rats
}

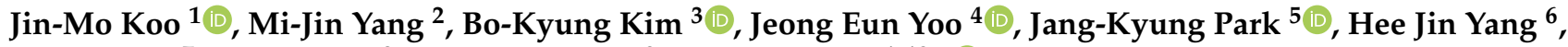 \\ Jongkil Joo ${ }^{7}$, Bo Sun Joo ${ }^{8}$, Jeong-Doo Heo ${ }^{9}$ and Ki-Tae Ha ${ }^{1,10, *[1]}$
}

check for

updates

Citation: Koo, J.-M.; Yang, M.-J.; Kim, B.-K.; Yoo, J.E.; Park, J.-K.; Yang, H.J.; Joo, J.; Joo, B.S.; Heo, J.-D.; Ha, K.-T. Acute and Repeated Toxicological Study of Anti-Inflammatory Herbal Formula, Yeosinsan, in Rats. Appl. Sci. 2021, 11, 9325. https://doi.org/ 10.3390/app11199325

Academic Editor: Giovanna Traina

Received: 8 September 2021

Accepted: 4 October 2021

Published: 8 October 2021

Publisher's Note: MDPI stays neutral with regard to jurisdictional claims in published maps and institutional affiliations.

Copyright: (c) 2021 by the authors Licensee MDPI, Basel, Switzerland. This article is an open access article distributed under the terms and conditions of the Creative Commons Attribution (CC BY) license (https:/ / creativecommons.org/licenses/by/ $4.0 /)$
1 Department of Korean Medicine, School of Korean Medicine, Pusan National University, Busandaehak-ro 49, Mulgeum-eup, Yangsan 50612, Korea; 201876804@pusan.ac.kr

2 Pathology Research Group, Jeonbuk Branch Institute, Kore Institute of Toxicology, Korea Research Institute of Chemical Technology, Baekhak 1-gil 30, Jeonbuk, Jeongeup 56212, Korea; mjyang@kitox.re.kr

3 General Toxicology Research Group, Jeonbuk Branch Institute, Kore Institute of Toxicology, Korea Research Institute of Chemical Technology, Baekhak 1-gil 30, Jeonbuk, Jeongeup 56212, Korea; kimbo@kitox.re.kr

4 Department of Korean Medical Gynecology, College of Korean Medicine, Daejeon University, Daehak-ro 62, Dong-gu, Daejeon 34520, Korea; jeyoo@dju.ac.kr

5 Department of Korean Medicine Obstetrics and Gynecology, School of Korean Medicine, Pusan National University, Geumo-ro 20, Mulgeum-eup, Yangsan 50612, Korea; vivat314@pusan.ac.kr

6 CHO-A Pharm. Co., Ltd., Dangsan-ro 2 gil 12, Yeongdeungpo-gu, Seoul 07299, Korea; hjyang@choa.co.kr

7 Department of Obstetrics and Gynecology, School of Medicine, Pusan National University Hospital, Gudeok-ro 179, Seo-gu, Busan 49214, Korea; jkjoo@pusan.ac.kr

8 Infertility Institute, Pohang Women's Hospital, Gyeongbuk, Pohang 37754, Korea; bosunjoo@hanmail.net

9 Bioenvironmental Science \& Toxicology Division, Gyeongnam Branch Institute, Korea Institute of Toxicology, Jegok-gil 17, Munsan-eup, Jinju 52834, Korea; jdher@kitox.re.kr

10 Korean Medical Research Center for Healthy Aging, Pusan National University, Busandaehak-ro 49, Mulgeum-eup, Yangsan 50612, Korea

* Correspondence: hagis@pusan.ac.kr; Tel.: +82-51-510-8464

Abstract: Yeosinsan, an herbal formula composed of roots of Paeonia lactiflora and tubers of Cyperus rotundus, was reported as a possible anti-inflammatory and pro-fertility drug. However, the safety of Yeosinsan has not yet been previously investigated. The possible acute and chronic oral toxicity of Yeosinsan was estimated using female and male Sprague Dawley rats. During the administration period, rats were monitored for mortality, body weight changes, food intake, clinical signs, and gross findings. Hematological analysis, serum biochemistry analysis, urinalysis, organ weight measurement, and histopathological examination were also conducted after sacrifice. Acute and chronic oral administration of Yeosinsan did not result in any signs of toxicity in the animals during the observation period. However, in the histopathological examination, several significant changes were observed in the stomach and spleen. In the high-dose group, extramedullary hematopoiesis and increased pigmentation in the spleen and squamous cell hyperplasia in the forestomach were observed. In conclusion, the no observed adverse effect level (NOAEL) of the test material, Yeosinsan, was estimated at $1000 \mathrm{mg} \cdot \mathrm{kg}^{-1} \cdot \mathrm{d}^{-1}$ for both male and female rats. Therefore, our data suggest that Yeosinsan might be safe to use for treating female infertility.

Keywords: Yeosinsan; Paeonia lactiflora; Cyperus rotundus; safety; oral toxicity

\section{Introduction}

Although the development of assisted reproduction technologies in recent decades has improved the outcomes of infertility treatment, a poor ovarian response and repetitive implantation failure are still major issues in female infertility [1,2]. Because there are very limited options for improving ovarian function and endometrial receptivity [3], many researchers have struggled to find novel therapeutic agents. Traditional medicines have been used for treating female infertility in many countries such as Korea, Japan, and China [4]. The effects of traditional herbal formulas, such as BaelanChagsangBang, Zhuyun 
recipe, Erbuzhuyu decoction, Dingkun Pill, and Wenshen Yangxue decoction, were previously studied [5-9]. Several studies have focused on ovarian dysfunction and defective endometrial receptivity as the molecular mechanism of these herbal remedies [10,11].

Paeonia lactiflora Pall. (Paeoniae Radix) and its major ingredient, paeoniflorin, have anti-inflammatory effects on several diseases, such as rheumatoid arthritis, inflammatory bowel disease, psoriasis, and asthma [12,13]. Cyperus rotundus L. (Cyperi Rhizoma) is also used for anti-oxidative and anti-inflammatory purposes [14,15]. In our previous study, the roots of $P$. lactiflora and the tubers of $C$. rotundus were able to treat female infertility by enhancing endometrial receptivity $[16,17]$. The possible effect of $P$. lactiflora on ovarian function was also reported in clinical studies on patients with polycystic ovarian disease [18-20]. In addition, Yeosinsan, an herbal formula composed of these two herbal medicines, was traditionally used for inflammation-related uterine bleeding and reported as a possible drug for improving female fertility that activates ovarian function and endometrial receptivity [21]. However, in the case of safety experiments, there are no reports on good laboratory practice (GLP)-level safety experiments of these herbal formulas, including BaelanChagsangBang, Zhuyun recipe, Erbuzhuyu decoction, Dingkun Pill, and Wenshen Yangxue decoction. In addition, very limited previous reports were found on the safety of these herbal medicines, including P. lactiflora and C. rotundus, or Yeosinsan $[14,22]$.

Thus, in this study, we evaluated the possible acute and chronic oral toxicity of Yeosinsan using a GLP rat model according to standard toxicology protocols. The safety data for Yeosinsan might play a crucial role in the approval of an investigational new drug application and in the protection of human subjects in clinical trials.

\section{Materials and Methods}

\subsection{Preparation of Yeosinsan Extract}

The extract of the herbal formula, Yeosinsan, was supplied by Cho-A Pharmaceutical Co. (Seoul, Korea). The Yeosinsan extract was prepared at a GMP-level facility at Cho-A Pharmaceutical Co., according to our previous protocol [21], and stored at the company under the codename CHOA_PLCR. Briefly, $4 \mathrm{~kg}$ of the two mixed herbal medicines $(P$. lactiflora and roasted C. rotundus with aqueous alcohol) was boiled in distilled water. The hGMP-level product of herbs according to the Ministry of Food and Drug Safety (MFDS) guideline was purchased from Green Myungpum Pharm (Namyangju, Korea), collected at Gyeongbuk province, Korea in 2019. The voucher specimens were deposited in the herbarium of Cho-A Pharmaceutical Co. The extract was concentrated and converted to a powder via a spray-drying process. The weight of the final extracted powder was $392 \mathrm{~g}$ (yield $=9.8 \%$ ). After the powder was dissolved in distilled water, it was filtered and stored until use.

\subsection{Animals}

The preclinical safety experiment was conducted by the Korea Institute of Toxicology (KIT, Daejeon, Korea), according to the guideline for the Care and Use of Laboratory Animal and the good laboratory practice (GLP) guideline (2019) of the MFDS, Government of Korea. All experimental designs were approved by the Institutional Animal Care and Use Committee (KIT experiment protocol number G217055 and G218038). KIT was approved by the Association for Assessment and Accreditation of Laboratory Animal Care International in 1998. Healthy animals were used for this test, and inspection reports provided by the animal suppliers were maintained as basic data. The animals selected in this study did not show clinical symptoms such as illness or injury and presented appropriate body weights. Sprague Dawley (SD) rats with a body weight of 189.2 to $216.3 \mathrm{~g}$ for males and 140.0 to $170.4 \mathrm{~g}$ for females were supplied by Orient Bio (Sungnam, Korea). Based on the most recent body weight, the Pristima System (Xybion Co., Lawrenceville, NJ) was used to randomly assign the rats to control and administration groups. The environment was automatically controlled according to standard working conditions (temperature: $23 \pm 3^{\circ} \mathrm{C}$, 
relative humidity: 30-70\%, 12-h light/12-h dark cycle, illumination: 150-300 lux, ventilation: 10-20 times per hour). The temperature and relative humidity were automatically monitored and recorded. At the end of experiments, animals were fasted for $12 \mathrm{~h}$ and euthanized with an inhalation of isoflurane (JSK, Goyang, Korea).

\subsection{Acute Oral Toxicity}

To investigate the acute oral toxicity of Yeosinsan in rats, this test was conducted using a single oral dose of $0,500,1000$, or $2000 \mathrm{mg} / \mathrm{kg}$ in each of the five male and female groups. The experimental animals were monitored for mortality, body weight changes, food intake, clinical signs, and gross findings during the administration period. Yeosinsan extract was administered once, and the observation period was 15 days after administration.

\subsection{Chronic Toxicity Test}

Yeosinsan was administered to male and female SD rats at oral doses of 0, 500, 1000, and $2000 \mathrm{mg} \cdot \mathrm{kg}^{-1} \cdot \mathrm{d}^{-1}$. The experimental animals were monitored for mortality, body weight changes, food intake, clinical signs, and gross findings during the administration period. Organ weight, histopathological, hematological, and biochemical analyses were also conducted after sacrifice. Yeosinsan extract was administered once a day during every observation day. The observation period was 92 days for males and 93 days for females.

\subsection{Hematology and Serum Biochemical Analysis}

For hematological analysis, total leukocyte count (WBC), mean corpuscular hemoglobin $(\mathrm{MCH})$, total red blood cell count (RBC), mean corpuscular hemoglobin concentration (MCHC), hemoglobin (HGB), platelet count (PLT), hematocrit (HCT), reticulocyte count, mean corpuscular volume (MCV), and WBC differential counts were measured using an ADVIA 2120i hematology analyzer (Siemens, Munich, Germany). Prothrombin time (PT) and activated partial thromboplastin time (APTT) were measured using an ACL Elite Pro Coagulation Analyzer (Instrumentation Laboratory, Bedford, MA). About $1.5 \mathrm{~mL}$ of blood was collected from each animal. About $0.5 \mathrm{~mL}$ of blood was collected in a blood collection tube containing an anticoagulant (EDTA-2K) for hematological analysis. About $1.0 \mathrm{~mL}$ of blood was collected in a blood collection tube containing 3.2\% sodium citrate. The plasma was separated by centrifugation (approximately $3000 \mathrm{rpm}$ for $10 \mathrm{~min}$ at room temperature), and a blood clotting time test was conducted.

For serum biochemical analysis, glucose (GLU), alanine aminotransferase (ALT), blood urea nitrogen (BUN), total bilirubin (TBIL), creatinine (CREA), alkaline phosphatase (ALP), total protein (TP), gamma-glutamyl transpeptidase (GGT), albumin (ALB), creatine phosphokinase $(\mathrm{CK})$, the albumin/globulin ratio $(\mathrm{A} / \mathrm{G})$, calcium $(\mathrm{Ca})$, total cholesterol (TCHO), inorganic phosphorus (IP), triglyceride (TG), sodium (Na), phospholipid (PL), potassium $(\mathrm{K})$, aspartate aminotransferase (AST), and chloride $(\mathrm{Cl})$ were measured using a TBA120FR chemistry analyzer (Toshiba Co., Tokyo, Japan). Before blood collection, all animals were fasted for more than $16 \mathrm{~h}$. About $1.5 \mathrm{~mL}$ of blood was collected in a tube without anticoagulants. After incubation at room temperature for at least $90 \mathrm{~min}$, the serum was separated by centrifugation (approximately $3000 \mathrm{rpm}$ for $10 \mathrm{~min}$ at room temperature).

\subsection{Urinalysis}

For urinalysis, urine volume (VOL) was measured by reading the scales on the urine collection tube. Clarity (CLA) was examined visually. After visual examination, the data were input into Cobas U411. Glucose (GLU), color (COL), erythrocyte (ERY), pH, ketone (KETu), specific gravity (SG), leukocytes (LEU), bilirubin (BILu), protein (PRO), urobilinogen (UBG), and nitrite (NIT) were measured using a Cobas U411 urine analyzer (Roche, Germany) and Combur 10 TM urine sticks (Roche, Germany). Urine potassium (UK), chloride (UCl), and sodium (UNa) were measured using a TBA 120FR automated chemistry analyzer (Toshiba, Japan). Urine was collected from all animals for about $16 \mathrm{~h}$ within the week before necropsy. 


\subsection{Necropsy and Histopathology}

After the test period, all remaining animals were euthanized using $\mathrm{CO}_{2}$ inhalation. A full detailed gross necropsy was conducted for each dose group. The weights of the following organs were measured for all animals: brain, kidneys, pituitary gland, adrenal glands, liver, testes, spleen, epididymis, heart, lung, thymus, thyroids (with parathyroids), salivary glands (submandibular and sublingual), uterus (with cervix), seminal vesicles (with coagulation gland), ovaries, and prostate. Reference samples were saved for all animals and visible abnormalities in the control group and highest dose group, and histopathological examination was conducted after hematoxylin and eosin staining. Reference samples were also saved for the 500 and $1000 \mathrm{mg} \cdot \mathrm{kg}^{-1} \cdot \mathrm{d}^{-1}$ dose groups for the stomach and spleen, where changes due to the test substance were observed, and histopathological examination was conducted.

\subsection{Statistical Analysis}

The data collected during the test period are presented as the inter-group mean and standard deviation. The data were statistically analyzed using the Pristima System (Xybion Co., Lawrenceville, NJ, USA). The inter-group comparison of the control and administration groups was conducted using a multiple comparison analysis. The parameters tested included body weight, body weight gain, food consumption, hematology, coagulation, clinical chemistry, urinalysis, and organ weight. The data were tested for equal variances using Bartlett's test, the equally distributed data were tested using one-way analysis of variance, and the differences between groups were analyzed using Dunnett's test. The least significant difference (LSD) was set as $P<0.05$ in all statistical analyses.

\section{Results}

\subsection{Acute Oral Toxicity}

The acute oral administration of Yeosinsan extract (single oral dose of 500, 1000, and $2000 \mathrm{mg} / \mathrm{kg}$ ) did not result in any signs of toxicity in the animals during the 15 days of the observation period (Table 1). Regarding body weight, no weight changes associated with the test substance were observed in any dose group (Figure 1). In the microscopic observations, no changes associated with the test substance were observed in any dose group (Table S1). Regarding mortality, no dead animals were observed during the test period (Table S2).

Table 1. Summary of clinical signs of acute oral toxicity.

\begin{tabular}{|c|c|c|c|c|c|c|}
\hline \multicolumn{3}{|c|}{ Dosage Group (mg/kg) } & 0 & 500 & 1000 & 2000 \\
\hline \multirow{3}{*}{ Male } & \multirow{3}{*}{$\begin{array}{c}\text { No abnormalities } \\
\text { detected }\end{array}$} & \multirow{3}{*}{$\begin{array}{c}\text { Number of animals examined } \\
\text { Number of animals not affected } \\
\text { Mean number of animal days with no } \\
\text { clinical sign }\end{array}$} & 5 & 5 & 5 & 5 \\
\hline & & & 5 & 5 & 5 & 5 \\
\hline & & & 15 & 15 & 15 & 15 \\
\hline \multirow{3}{*}{ Female } & \multirow{3}{*}{$\begin{array}{c}\text { No abnormalities } \\
\text { detected }\end{array}$} & Number of animals examined & 5 & 5 & 5 & 5 \\
\hline & & Number of animals not affected & 5 & 5 & 5 & 5 \\
\hline & & $\begin{array}{c}\text { Mean number of animal days with no } \\
\text { clinical sign }\end{array}$ & 15 & 15 & 15 & 15 \\
\hline
\end{tabular}



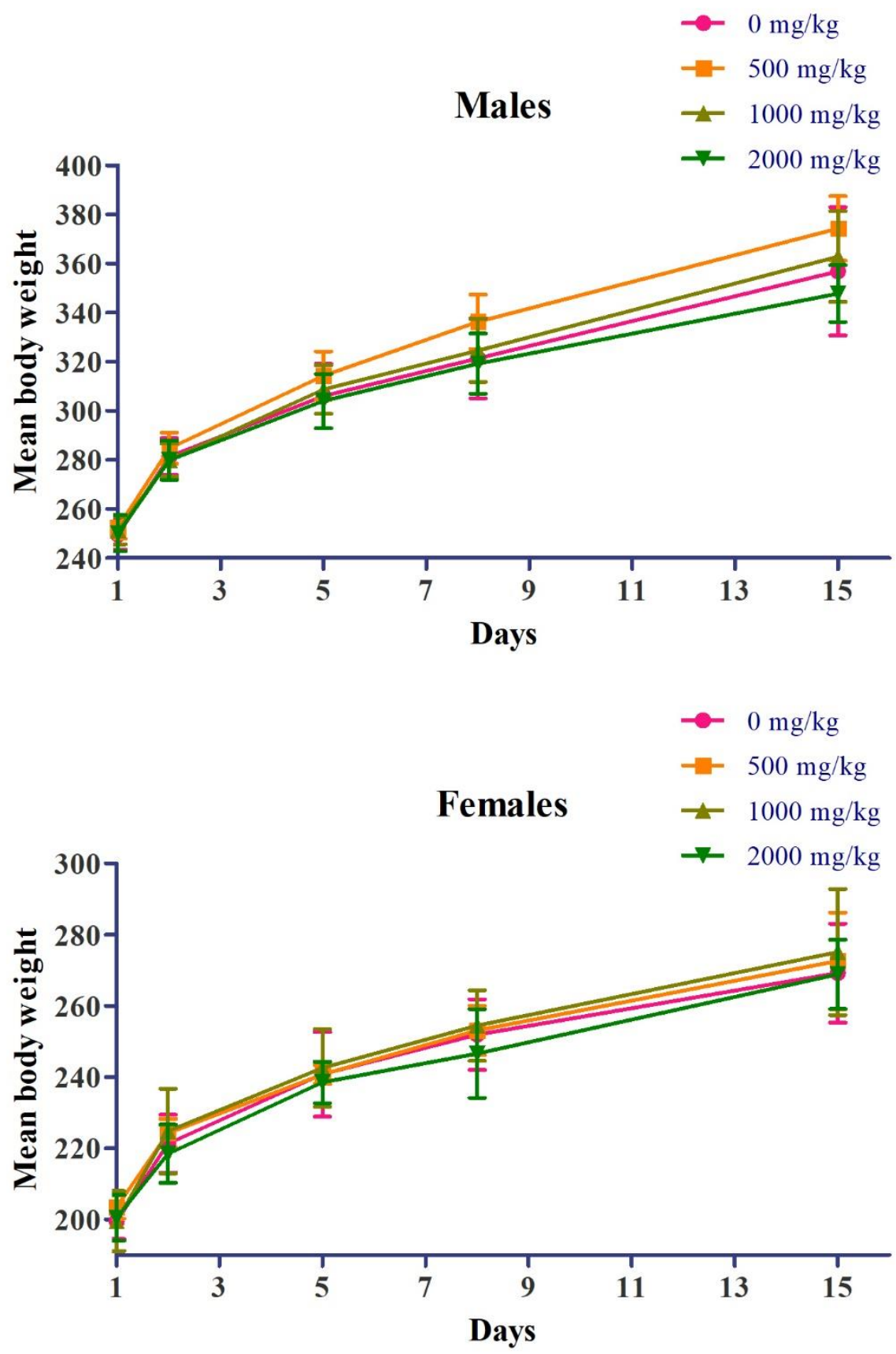

Figure 1. Effects of acute oral administration of Yeosinsan extract on body weight over 15 days. Body weights of male and female rats orally exposed to Yeosinsan extract at 0, 500, 1000, and $2000 \mathrm{mg} / \mathrm{kg}$. Data are expressed as the mean $\pm S D, n=5$ animals per group.

\subsection{Chronic oral Toxicity}

\subsubsection{Clinical Signs and Mortality}

The chronic oral administration of Yeosinsan extract did not result in any signs of toxicity in the animals during the observation period (92 days for males and 93 days for females). In the male control group, in one subject (animal no. 1), loss of fur and scabbing were observed from day 28 to day 36 after administration. In the female $500 \mathrm{mg} \cdot \mathrm{kg}^{-1} \cdot \mathrm{d}^{-1}$ dose group, in two subjects (animal no. 51 and 53), loss of fur was observed from 61 days (posterior cranium) and 71 days (right upper forelimb) to 93 days after administration. In the female $2000 \mathrm{mg} \cdot \mathrm{kg}^{-1} \cdot \mathrm{d}^{-1}$ dose group, in two subjects (animal no. 79 and 80), loss of fur was observed from 71 days (animal no. 79, right neck) and 82 days (animal no. 80, left lumbar region) to 93 days after administration. These symptoms were considered to be 
the result of repeated physical stimulation during the animal handling process for oral administration (Table 2).

Table 2. Summary of clinical signs of chronic oral toxicity.

\begin{tabular}{|c|c|c|c|c|c|c|}
\hline & Dosage Grou & $\left(\mathrm{mg} \cdot \mathrm{kg}^{-1} \cdot \mathrm{d}^{-1}\right)$ & 0 & 500 & 1000 & 2000 \\
\hline \multirow{9}{*}{ Male } & \multirow{3}{*}{$\begin{array}{l}\text { No abnormalities } \\
\text { detected }\end{array}$} & Number of animals examined & 10 & 10 & 10 & 10 \\
\hline & & Number of animals not affected & 10 & 10 & 10 & 10 \\
\hline & & $\begin{array}{c}\text { Mean number of animal days with no } \\
\text { clinical sign }\end{array}$ & 91.10 & 92.00 & 92.00 & 92.00 \\
\hline & \multirow{3}{*}{$\begin{array}{l}\text { Fur, hair, coat } \\
\text { Loss of fur, posterior } \\
\text { neck, slight }\end{array}$} & Number of animals examined & 10 & 10 & 10 & 10 \\
\hline & & Number of animals affected & 1 & 0 & 0 & 0 \\
\hline & & $\begin{array}{c}\text { Mean number of animal days with } \\
\text { clinical sign }\end{array}$ & 9.00 & 0.00 & 0.00 & 0.00 \\
\hline & \multirow{3}{*}{$\begin{array}{c}\text { Skin } \\
\text { Scab, posterior } \\
\text { neck, slight }\end{array}$} & Number of animals examined & 10 & 10 & 10 & 10 \\
\hline & & Number of animals affected & 1 & 0 & 0 & 0 \\
\hline & & $\begin{array}{c}\text { Mean number of animal days with } \\
\text { clinical sign }\end{array}$ & 9.00 & 0.00 & 0.00 & 0.00 \\
\hline \multirow{15}{*}{ Female } & \multirow{3}{*}{$\begin{array}{l}\text { No abnormalities } \\
\text { detected }\end{array}$} & Number of animals examined & 10 & 10 & 10 & 10 \\
\hline & & Number of animals not affected & 10 & 10 & 10 & 10 \\
\hline & & $\begin{array}{c}\text { Mean number of animal days with no } \\
\text { clinical sign }\end{array}$ & 93.00 & 86.40 & 93.00 & 89.50 \\
\hline & \multirow{3}{*}{$\begin{array}{l}\text { Fur, hair, coat } \\
\text { Loss of fur, left lumbar } \\
\text { region, slight }\end{array}$} & Number of animals examined & 10 & 10 & 10 & 10 \\
\hline & & Number of animals affected & 0 & 0 & 0 & 1 \\
\hline & & $\begin{array}{c}\text { Mean number of animal days with } \\
\text { clinical sign }\end{array}$ & 0.00 & 0.00 & 0.00 & 12.00 \\
\hline & \multirow{3}{*}{$\begin{array}{l}\text { Loss of fur, posterior } \\
\text { cranium, slight }\end{array}$} & Number of animals examined & 10 & 10 & 10 & 10 \\
\hline & & Number of animals affected & 0 & 2 & 0 & 0 \\
\hline & & $\begin{array}{c}\text { Mean number of animal days with } \\
\text { clinical sign }\end{array}$ & 0.00 & 33.00 & 0.00 & 0.00 \\
\hline & \multirow{3}{*}{$\begin{array}{l}\text { Loss of fur, right } \\
\text { neck, slight }\end{array}$} & Number of animals examined & 10 & 10 & 10 & 10 \\
\hline & & Number of animals affected & 0 & 0 & 0 & 1 \\
\hline & & $\begin{array}{c}\text { Mean number of animal days with } \\
\text { clinical sign }\end{array}$ & 0.00 & 0.00 & 0.00 & 23.00 \\
\hline & \multirow{3}{*}{$\begin{array}{l}\text { Loss of fur, right upper } \\
\text { forelimb, slight }\end{array}$} & Number of animals examined & 10 & 10 & 10 & 10 \\
\hline & & Number of animals affected & 0 & 2 & 0 & 0 \\
\hline & & $\begin{array}{c}\text { Mean number of animal days with } \\
\text { clinical sign }\end{array}$ & 0.00 & 23.00 & 0.00 & 0.00 \\
\hline
\end{tabular}

Regarding mortality, no dead animals were observed during the test period (Table S3).

\subsubsection{Body Weight and Feed Intake}

Regarding body weight, in the female $2000 \mathrm{mg} \cdot \mathrm{kg}^{-1} \cdot \mathrm{d}^{-1}$ dose group (G4), weight loss was observed, which was statistically significant compared to that in the control group (G1), at 8 days and 36 days after administration (Figure 2). Regarding food consumption, in the female $1000 \mathrm{mg} \cdot \mathrm{kg}^{-1} \cdot \mathrm{d}^{-1}$ dose group (G3), there was a decrease in food consumption, which was statistically significant compared to that in the control group (G1), at 78 days after administration. In the female $2000 \mathrm{mg} \cdot \mathrm{kg}^{-1} \cdot \mathrm{d}^{-1}$ dose group (G4), there was a decrease 
in food consumption, which was statistically significant compared to that in the control group (G1), at 8 days after administration (Figure 3).

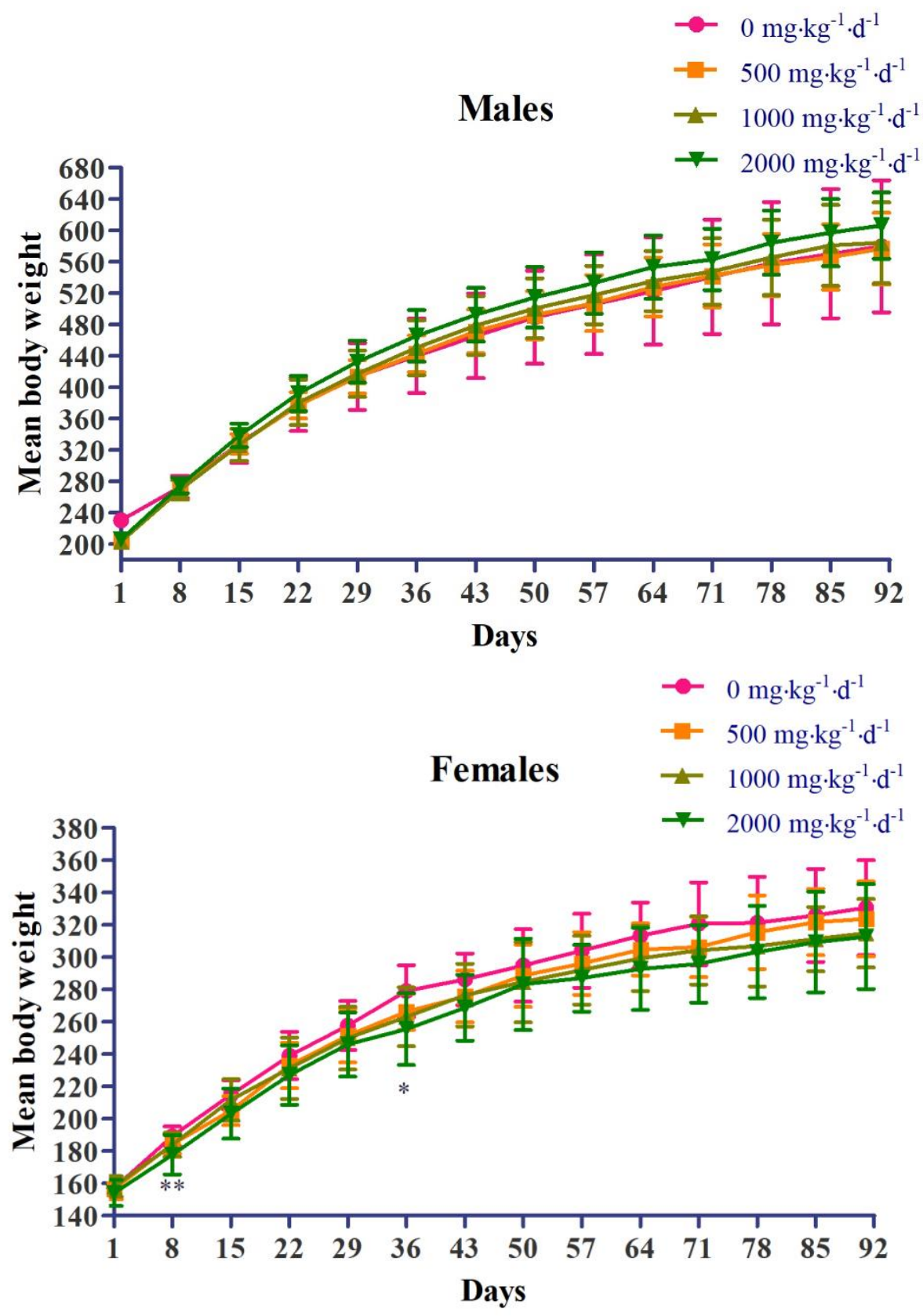

Figure 2. Effects of chronic oral administration of Yeosinsan extract on body weight for 91 days. In the female $2000 \mathrm{mg} \cdot \mathrm{kg}^{-1} \cdot \mathrm{d}^{-1}$ dose group, the weight loss observed was statistically significant compared to that in the control group at 8 days and 36 days after administration. Data are expressed as the mean $\pm \mathrm{SD}, \mathrm{n}=10$ animals per group. ${ }^{*} P<0.05,{ }^{* *} P<0.01$ according to Dunnett's LSD test. 


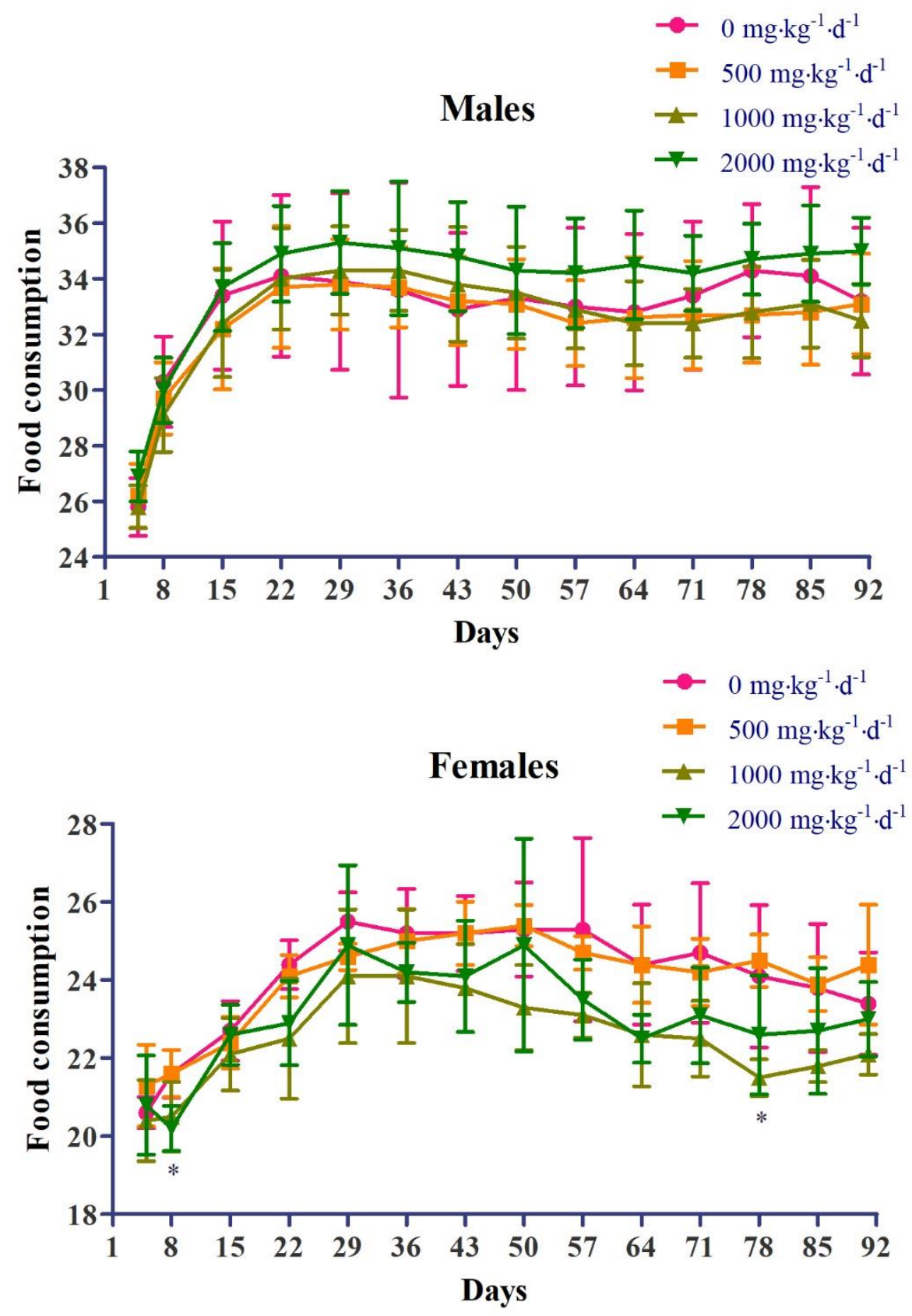

Figure 3. Effects of chronic oral administration of Yeosinsan extract on food consumption for 91 days. In the female $1000 \mathrm{mg} \cdot \mathrm{kg}^{-1} \cdot \mathrm{d}^{-1}$ dose group, a decrease in food consumption was observed, which was statistically significant compared to that in the control group at 78 days after administration. In the female $2000 \mathrm{mg} \cdot \mathrm{kg}^{-1} \cdot \mathrm{d}^{-1}$ dose group, a decrease in food consumption was observed, which was statistically significant compared to that in the control group at 8 days after administration. Data are expressed as the mean $\pm \mathrm{SD}, \mathrm{n}=4$ cages per group. ${ }^{*} P<0.05$ according to Dunnett's LSD test.

\subsubsection{Organ Weight}

Regarding the absolute organ weight, in the male $2000 \mathrm{mg} \cdot \mathrm{kg}^{-1} \cdot \mathrm{d}^{-1}$ dose group, the absolute and relative weight gain of the kidneys (1.18 and 1.13 times, respectively) and the relative weight loss of the epididymis ( 0.87 times) were statistically significant. In the female dose groups at or over $1000 \mathrm{mg} \cdot \mathrm{kg}^{-1} \cdot \mathrm{d}^{-1}$, relative weight gain was observed in the spleen (maximum 1.22 times). These changes were considered to be unrelated to the test substance because either histological changes were not observed or the changes were caused by specific objects (Table 3, Table S4). 
Table 3. Effects of chronic oral administration of Yeosinsan extract on absolute organ weights.

\begin{tabular}{|c|c|c|c|c|c|}
\hline Dosa & Group $\left(\mathrm{mg} \cdot \mathrm{kg}^{-1} \cdot \mathrm{d}^{-1}\right)$ & 0 & 500 & 1000 & 2000 \\
\hline \multirow{16}{*}{ Male } & TBW $(\mathrm{g})$ & $546.1 \pm 79.76$ & $543.6 \pm 42.11$ & $551.0 \pm 49.88$ & $565.0 \pm 42.30$ \\
\hline & (ADRG) Adrenal glands (g) & $0.062 \pm 0.0096$ & $0.063 \pm 0.0072$ & $0.065 \pm 0.0110$ & $0.064 \pm 0.0042$ \\
\hline & (BRA) Brain $(\mathrm{g})$ & $2.163 \pm 0.1352$ & $2.214 \pm 0.0803$ & $2.174 \pm 0.0824$ & $2.214 \pm 0.0878$ \\
\hline & (HT) Heart $(\mathrm{g})$ & $1.706 \pm 0.2359$ & $1.584 \pm 0.1162$ & $1.641 \pm 0.1242$ & $1.679 \pm 0.1135$ \\
\hline & (KID) Kidneys (g) & $3.625 \pm 0.3945$ & $3.958 \pm 0.3891$ & $4.065 \pm 0.4607$ & $4.280 \pm 0.4100^{* *}$ \\
\hline & (LIV) Liver (g) & $15.464 \pm 3.4509$ & $15.848 \pm 2.1123$ & $15.676 \pm 2.0249$ & $16.580 \pm 1.3323$ \\
\hline & (PITG) Pituitary glands (g) & $0.014 \pm 0.0033$ & $0.015 \pm 0.0025$ & $0.013 \pm 0.0016$ & $0.013 \pm 0.0015$ \\
\hline & (SPL) Spleen (g) & $0.925 \pm 0.2494$ & $0.903 \pm 0.1138$ & $0.916 \pm 0.0730$ & $1.068 \pm 0.2334$ \\
\hline & (TES) Testes (g) & $3.517 \pm 0.3382$ & $3.515 \pm 0.3207$ & $3.516 \pm 0.2252$ & $3.319 \pm 0.7046$ \\
\hline & (THY) Thymus (g) & $0.478 \pm 0.1020$ & $0.446 \pm 0.1158$ & $0.449 \pm 0.0757$ & $0.463 \pm 0.1338$ \\
\hline & (SALG) Salivary glands (g) & $0.754 \pm 0.1519$ & $0.714 \pm 0.0505$ & $0.765 \pm 0.0780$ & $0.783 \pm 0.0553$ \\
\hline & (EPI) Epididymis (g) & $1.603 \pm 0.1721$ & $1.610 \pm 0.1146$ & $1.520 \pm 0.0698$ & $1.453 \pm 0.2286$ \\
\hline & (LUN) Lung $(\mathrm{g})$ & $1.721 \pm 0.1855$ & $1.772 \pm 0.1125$ & $1.714 \pm 0.0862$ & $1.836 \pm 0.0848$ \\
\hline & $\begin{array}{l}\text { (THPA) Thyroid and } \\
\text { parathyroid glands (g) }\end{array}$ & $0.024 \pm 0.0058$ & $0.028 \pm 0.0069$ & $0.023 \pm 0.0028$ & $0.025 \pm 0.0043$ \\
\hline & $\begin{array}{l}\text { (SVCG) Seminal vesicles } \\
\text { with coagulating gland }\end{array}$ & $1.983 \pm 0.4411$ & $1.877 \pm 0.4002$ & $1.805 \pm 0.2810$ & $1.702 \pm 0.3097$ \\
\hline & (PROS) Prostate (g) & $0.606 \pm 0.1249$ & $0.613 \pm 0.1515$ & $0.581 \pm 0.1695$ & $0.621 \pm 0.1494$ \\
\hline \multirow{14}{*}{ Female } & TBW $(\mathrm{g})$ & $309.4 \pm 26.33$ & $300.5 \pm 20.04$ & $292.2 \pm 19.22$ & $290.4 \pm 29.27$ \\
\hline & (ADRG) Adrenal glands (g) & $0.081 \pm 0.0062$ & $0.082 \pm 0.0129$ & $0.078 \pm 0.0084$ & $0.077 \pm 0.0154$ \\
\hline & (BRA) Brain (g) & $1.997 \pm 0.0653$ & $2.019 \pm 0.0769$ & $1.987 \pm 0.0634$ & $1.969 \pm 0.0583$ \\
\hline & (HT) Heart (g) & $1.046 \pm 0.0966$ & $1.054 \pm 0.0735$ & $0.974 \pm 0.0904$ & $0.964 \pm 0.0835$ \\
\hline & (KID) Kidneys (g) & $2.321 \pm 0.2309$ & $2.303 \pm 0.1974$ & $2.246 \pm 0.1541$ & $2.297 \pm 0.2777$ \\
\hline & (LIV) Liver (g) & $8.729 \pm 1.1196$ & $8.443 \pm 0.7715$ & $8.377 \pm 0.4921$ & $8.611 \pm 0.9457$ \\
\hline & (OVA) Ovaries (g) & $0.098 \pm 0.0249$ & $0.095 \pm 0.0156$ & $0.084 \pm 0.0139$ & $0.093 \pm 0.0197$ \\
\hline & (PITG) Pituitary glands (g) & $0.021 \pm 0.0043$ & $0.018 \pm 0.0032$ & $0.019 \pm 0.0036$ & $0.020 \pm 0.0041$ \\
\hline & (SPL) Spleen (g) & $0.574 \pm 0.0379$ & $0.611 \pm 0.1084$ & $0.657 \pm 0.0855$ & $0.661 \pm 0.1035$ \\
\hline & (THY) Thymus (g) & $0.343 \pm 0.0581$ & $0.329 \pm 0.0683$ & $0.331 \pm 0.0479$ & $0.344 \pm 0.0899$ \\
\hline & (SALG) Salivary glands (g) & $0.466 \pm 0.0690$ & $0.458 \pm 0.0476$ & $0.449 \pm 0.0369$ & $0.471 \pm 0.0413$ \\
\hline & (LUN) Lung (g) & $1.347 \pm 0.1355$ & $1.346 \pm 0.0768$ & $1.329 \pm 0.0896$ & $1.344 \pm 0.1263$ \\
\hline & $\begin{array}{l}\text { (THPA) Thyroid and } \\
\text { parathyroid glands (g) }\end{array}$ & $0.019 \pm 0.0039$ & $0.020 \pm 0.0037$ & $0.019 \pm 0.0025$ & $0.019 \pm 0.0036$ \\
\hline & (UTEC) Uterus / cervix & $0.725 \pm 0.1570$ & $0.903 \pm 0.4507$ & $0.741 \pm 0.3030$ & $0.741 \pm 0.3344$ \\
\hline
\end{tabular}

Data are expressed as the mean $\pm \mathrm{SD}, \mathrm{n}=10$ animals per group. ${ }^{* *} p<0.01$ according to Dunnett's LSD test.

\subsubsection{Hematological Test}

In the hematological examination, in the female $500 \mathrm{mg} \cdot \mathrm{kg}^{-1} \cdot \mathrm{d}^{-1}$ and all groups at or over $1000 \mathrm{mg} \cdot \mathrm{kg}^{-1} \cdot \mathrm{d}^{-1}$ (G3, G4), a decrease (minimum 0.90 times) in RBC and an increase (maximum 1.49 times and 1.58 times, respectively) in the absolute and relative reticulocyte count (RETA and RET\%) were observed. In the $2000 \mathrm{mg} \cdot \mathrm{kg}^{-1} \cdot \mathrm{d}^{-1}$ dose group (G4), an increase (maximum 1.07 times) in MCV and MCH was observed (Table 4). In the $2000 \mathrm{mg} \cdot \mathrm{kg}^{-1} \cdot \mathrm{d}^{-1}$ dose group (G4), an increase (maximum 1.20 times) in PT was observed. These changes were considered to have no toxicological significance because either the values were within the physiologically normal range or associated findings were not observed in the histopathological examination. The other statistically significant changes observed were not associated with the test substance because of a lack of dose correlation, a slight difference from the control group (G1), or values within the physiologically normal range (Table 5). 
Table 4. Effects of chronic oral administration of Yeosinsan extract on hematology.

\begin{tabular}{|c|c|c|c|c|c|}
\hline \multicolumn{2}{|c|}{ Dosage Group $\left(\mathrm{mg} \cdot \mathrm{kg}^{-1} \cdot \mathrm{d}^{-1}\right)$} & \multirow{2}{*}{$\frac{\mathbf{0}}{9.06 \pm 0.626}$} & \multirow{2}{*}{$\frac{500}{8.70 \pm 0.371}$} & \multirow{2}{*}{$\frac{1000}{8.40 \pm 0.337^{* *}}$} & \multirow{2}{*}{$\frac{2000}{8.21 \pm 0.347^{* * *}}$} \\
\hline \multirow{22}{*}{ Male } & $\operatorname{RBC}\left(\times 10^{6} / \mu \mathrm{L}\right)$ & & & & \\
\hline & $\operatorname{HGB}(\mathrm{g} / \mathrm{dL})$ & $15.6 \pm 0.54$ & $15.3 \pm 0.37$ & $15.1 \pm 0.53$ & $15.2 \pm 0.41$ \\
\hline & HCT $(\%)$ & $50.8 \pm 1.96$ & $49.7 \pm 1.23$ & $48.9 \pm 2.14$ & $49.2 \pm 1.61$ \\
\hline & MCV (fL) & $56.2 \pm 2.71$ & $57.2 \pm 2.2$ & $58.3 \pm 1.78$ & $60.0 \pm 2.14^{* *}$ \\
\hline & $\mathrm{MCH}(\mathrm{pg})$ & $17.4 \pm 1.06$ & $17.7 \pm 0.8$ & $17.9 \pm 0.55$ & $18.6 \pm 0.65^{* *}$ \\
\hline & $\operatorname{MCHC}(\mathrm{g} / \mathrm{dL})$ & $30.8 \pm 0.68$ & $30.9 \pm 0.56$ & $30.8 \pm 0.5$ & $30.9 \pm 0.47$ \\
\hline & RET\% $\%(\%)$ & $2.03 \pm 0.453$ & $2.25 \pm 0.412$ & $2.76 \pm 0.366^{* *}$ & $3.21 \pm 0.352 * *$ \\
\hline & RETA $\left(\times 10^{9} / \mu \mathrm{L}\right)$ & $181.9 \pm 30.02$ & $194.1 \pm 28.71$ & $231.5 \pm 27.26^{* *}$ & $263.5 \pm 27.76^{* *}$ \\
\hline & $\operatorname{PLT}\left(\times 10^{5} / \mu \mathrm{L}\right)$ & $884.8 \pm 241.68$ & $996.4 \pm 95.96$ & $1036.3 \pm 148.98$ & $989.3 \pm 219.31$ \\
\hline & NEU\% $(\%)$ & $14.9 \pm 5.49$ & $16.5 \pm 5.01$ & $14.7 \pm 4.78$ & $16.1 \pm 5.57$ \\
\hline & LYM \% (\%) & $79.7 \pm 5.7$ & $78.2 \pm 5.45$ & $80.5 \pm 4.35$ & $78.6 \pm 6.26$ \\
\hline & EOS $\%(\%)$ & $1.2 \pm 0.49$ & $1.3 \pm 0.22$ & $1.1 \pm 0.28$ & $1 \pm 0.37$ \\
\hline & MON (\%) & $2.7 \pm 0.76$ & $2.4 \pm 0.77$ & $2.4 \pm 0.95$ & $2.5 \pm 0.85$ \\
\hline & BAS $\%(\%)$ & $0.4 \pm 0.09$ & $0.4 \pm 0.11$ & $0.4 \pm 0.13$ & $0.5 \pm 0.15$ \\
\hline & LUC\% $(\%)$ & $1.1 \pm 0.6$ & $1.3 \pm 0.65$ & $1 \pm 0.4$ & $1.3 \pm 0.67$ \\
\hline & $\mathrm{WBC}\left(\times 10^{3} / \mu \mathrm{L}\right)$ & $11.95 \pm 2.923$ & $10.76 \pm 2.25$ & $9.23 \pm 1.877$ & $10.35 \pm 2.063$ \\
\hline & $\operatorname{NEUA}\left(\times 10^{3} / \mu \mathrm{L}\right)$ & $1.76 \pm 0.75$ & $1.79 \pm 0.695$ & $1.32 \pm 0.372$ & $1.68 \pm 0.801$ \\
\hline & LYMA $\left(\times 10^{3} / \mu \mathrm{L}\right)$ & $9.55 \pm 2.582$ & $8.39 \pm 1.67$ & $7.47 \pm 1.768$ & $8.11 \pm 1.524$ \\
\hline & $\operatorname{MONA}\left(\times 10^{3} / \mu \mathrm{L}\right)$ & $0.32 \pm 0.107$ & $0.26 \pm 0.119$ & $0.22 \pm 0.085$ & $0.26 \pm 0.135$ \\
\hline & $\operatorname{EOSA}\left(\times 10^{3} / \mu \mathrm{L}\right)$ & $0.14 \pm 0.054$ & $0.14 \pm 0.047$ & $0.1 \pm 0.036$ & $0.1 \pm 0.04$ \\
\hline & BASA $\left(\times 10^{3} / \mu \mathrm{L}\right)$ & $0.05 \pm 0.012$ & $0.05 \pm 0.016$ & $0.04 \pm 0.016$ & $0.05 \pm 0.016$ \\
\hline & LUCA $\left(\times 10^{3} / \mu \mathrm{L}\right)$ & $0.14 \pm 0.089$ & $0.15 \pm 0.111$ & $0.09 \pm 0.043$ & $0.14 \pm 0.104$ \\
\hline \multirow{22}{*}{ Female } & $\mathrm{RBC}\left(\times 10^{6} / \mu \mathrm{L}\right)$ & $8.23 \pm 0.355$ & $7.91 \pm 0.236$ * & $7.91 \pm 0.285^{*}$ & $7.44 \pm 0.277^{* *}$ \\
\hline & HGB (g/dL) & $15.5 \pm 0.56$ & $15.2 \pm 0.61$ & $15.1 \pm 0.70$ & $14.8 \pm 0.39$ \\
\hline & HCT $(\%)$ & $49.2 \pm 1.61$ & $47.5 \pm 1.68$ & $47.9 \pm 2.43$ & $46.2 \pm 1.07^{* *}$ \\
\hline & MCV (fL) & $59.8 \pm 1.38$ & $60.0 \pm 1.04$ & $60.5 \pm 1.75$ & $62.1 \pm 0.96^{* *}$ \\
\hline & $\mathrm{MCH}(\mathrm{pg})$ & $18.9 \pm 0.36$ & $19.2 \pm 0.46$ & $19.0 \pm 0.57$ & $19.9 \pm 0.33^{* *}$ \\
\hline & $\mathrm{MCHC}(\mathrm{g} / \mathrm{dL})$ & $31.5 \pm 0.46$ & $31.9 \pm 0.67$ & $31.5 \pm 0.42$ & $32.1 \pm 0.32 *$ \\
\hline & RET\% (\%) & $2.10 \pm 0.367$ & $2.73 \pm 0.296^{* *}$ & $3.27 \pm 0.375^{* *}$ & $2.98 \pm 0.486^{* *}$ \\
\hline & RETA $\left(\times 10^{9} / \mu \mathrm{L}\right)$ & $173.3 \pm 30.99$ & $216.1 \pm 23.02 * *$ & $258.9 \pm 34.10^{* *}$ & $220.9 \pm 33.50 * *$ \\
\hline & $\operatorname{PLT}\left(\times 10^{5} / \mu \mathrm{L}\right)$ & $996.1 \pm 96.69$ & $1052.1 \pm 105.30$ & $1074.4 \pm 78.18$ & $1087.1 \pm 137.76$ \\
\hline & NEU\% $\%(\%)$ & $11.4 \pm 5.63$ & $12.8 \pm 2.93$ & $12.7 \pm 5.93$ & $12.3 \pm 4.25$ \\
\hline & LYM\% (\%) & $83.4 \pm 5.78$ & $81.1 \pm 4.41$ & $82.1 \pm 6.21$ & $82.8 \pm 4.90$ \\
\hline & EOS $\%(\%)$ & $1.3 \pm 0.39$ & $1.4 \pm 0.55$ & $1.1 \pm 0.26$ & $1.1 \pm 0.52$ \\
\hline & MON (\%) & $2.4 \pm 0.88$ & $2.9 \pm 1.32$ & $2.3 \pm 0.57$ & $2.1 \pm 0.60$ \\
\hline & BAS $\%(\%)$ & $0.4 \pm 0.15$ & $0.5 \pm 0.16$ & $0.6 \pm 0.21$ & $0.5 \pm 0.32$ \\
\hline & LUC\% $(\%)$ & $1.1 \pm 0.41$ & $1.4 \pm 0.59$ & $1.2 \pm 0.24$ & $1.3 \pm 0.53$ \\
\hline & $\mathrm{WBC}\left(\times 10^{3} / \mu \mathrm{L}\right)$ & $7.82 \pm 2.014$ & $7.85 \pm 1.463$ & $6.4 \pm 0.911$ & $8.26 \pm 2.354$ \\
\hline & $\operatorname{NEUA}\left(\times 10^{3} / \mu \mathrm{L}\right)$ & $0.88 \pm 0.478$ & $1.01 \pm 0.295$ & $0.79 \pm 0.331$ & $0.98 \pm 0.358$ \\
\hline & LYMA $\left(\times 10^{3} / \mu \mathrm{L}\right)$ & $6.54 \pm 1.910$ & $6.35 \pm 1.176$ & $5.28 \pm 0.998$ & $6.88 \pm 2.152$ \\
\hline & $\operatorname{MONA}\left(\times 10^{3} / \mu \mathrm{L}\right)$ & $0.19 \pm 0.068$ & $0.23 \pm 0.118$ & $0.15 \pm 0.035$ & $0.17 \pm 0.074$ \\
\hline & $\operatorname{EOSA}\left(\times 10^{3} / \mu \mathrm{L}\right)$ & $0.10 \pm 0.029$ & $0.12 \pm 0.063$ & $0.07 \pm 0.019$ & $0.09 \pm 0.053$ \\
\hline & $\mathrm{BASA}\left(\times 10^{3} / \mu \mathrm{L}\right)$ & $0.03 \pm 0.010$ & $0.04 \pm 0.013$ & $0.04 \pm 0.017$ & $0.04 \pm 0.017$ \\
\hline & LUCA $\left(\times 10^{3} / \mu \mathrm{L}\right)$ & $0.09 \pm 0.042$ & $0.11 \pm 0.055$ & $0.07 \pm 0.017$ & $0.1 \pm 0.043$ \\
\hline
\end{tabular}

Data are expressed as the mean $\pm \mathrm{SD}, \mathrm{n}=10$ animals per group. ${ }^{*} p<0.05,{ }^{* *} p<0.01$ according to Dunnett's LSD test.

Table 5. Effects of chronic oral administration of Yeosinsan extract on coagulation.

\begin{tabular}{|c|c|c|c|c|c|}
\hline \multicolumn{2}{|c|}{$\begin{array}{l}\text { Dosage Group } \\
\left(\mathrm{mg} \cdot \mathrm{kg}^{-1} \cdot \mathrm{d}^{-1}\right)\end{array}$} & \multirow{2}{*}{$\begin{array}{c}\mathbf{0} \\
15.7 \pm 1.36\end{array}$} & \multirow{2}{*}{$\begin{array}{c}\mathbf{5 0 0} \\
17.1 \pm 2.58\end{array}$} & \multirow{3}{*}{$\begin{array}{c}1000 \\
16.6 \pm 1.85 \\
17.5 \pm 0.91\end{array}$} & \multirow{3}{*}{$\begin{array}{c}2000 \\
18.8 \pm 2.06 \text { ** } \\
17.2 \pm 0.95\end{array}$} \\
\hline & $\mathrm{PT}(\mathrm{sec})$ & & & & \\
\hline Male & APTT (sec) & $17.2 \pm 1.10$ & $17.5 \pm 1.10$ & & \\
\hline \multirow{2}{*}{ Female } & $\mathrm{PT}(\mathrm{sec})$ & $13.8 \pm 0.65$ & $14.1 \pm 0.58$ & $14.2 \pm 0.81$ & $14.8 \pm 0.64$ * \\
\hline & APTT (sec) & $14.7 \pm 1.15$ & $15.4 \pm 1.35$ & $15.6 \pm 1.02$ & $15.6 \pm 0.79$ \\
\hline
\end{tabular}

Data are expressed as the mean $\pm \mathrm{SD}, \mathrm{n}=10$ animals per group. ${ }^{*} p<0.05,{ }^{* *} p<0.01$ according to Dunnett's LSD test. 


\subsubsection{Serum Biochemistry}

In the serum biochemistry examination, in the female dose groups at or over $1000 \mathrm{mg} \cdot \mathrm{kg}^{-1} \cdot \mathrm{d}^{-1}$, an increase (maximum 1.46 times) in TBIL was observed. In the female dose groups at or over $1000 \mathrm{mg} \cdot \mathrm{kg}^{-1} \cdot \mathrm{d}^{-1}$, an increase (maximum 1.46 times) in TBIL was observed. The other statistically significant changes observed were not associated with the test substance because of no dose correlation or a slight difference from the control group (G1) or physiologically normal range (Table 6).

Table 6. Summary of clinical chemistry of chronic oral toxicity.

\begin{tabular}{|c|c|c|c|c|c|}
\hline \multicolumn{2}{|c|}{ Dosage Group $\left(\mathrm{mg} \cdot \mathrm{kg}^{-1} \cdot \mathrm{d}^{-1}\right)$} & \multirow{2}{*}{$\begin{array}{c}\mathbf{0} \\
125.9 \pm 36.61\end{array}$} & \multirow{2}{*}{$\frac{500}{116.0 \pm 27.93}$} & \multirow{2}{*}{$\begin{array}{c}\mathbf{1 0 0 0} \\
111.0 \pm 17.16\end{array}$} & \multirow{2}{*}{$\begin{array}{c}\mathbf{2 0 0 0} \\
108.3 \pm 13.00\end{array}$} \\
\hline \multirow{20}{*}{ Male } & GUN (mg/dL) & & & & \\
\hline & $\mathrm{BUN}(\mathrm{mg} / \mathrm{dL})$ & $16.0 \pm 1.56$ & $15.8 \pm 2.62$ & $15.1 \pm 1.59$ & $16.9 \pm 1.56$ \\
\hline & CREA (mg/dL) & $0.48 \pm 0.043$ & $0.47 \pm 0.032$ & $0.46 \pm 0.020$ & $0.47 \pm 0.044$ \\
\hline & $\mathrm{TP}(\mathrm{g} / \mathrm{dL})$ & $6.65 \pm 0.297$ & $6.55 \pm 0.195$ & $6.53 \pm 0.339$ & $6.52 \pm 0.120$ \\
\hline & $\mathrm{ALB}(\mathrm{g} / \mathrm{dL})$ & $4.22 \pm 0.144$ & $4.21 \pm 0.101$ & $4.22 \pm 0.140$ & $4.23 \pm 0.091$ \\
\hline & A/G (ratio) & $1.74 \pm 0.102$ & $1.80 \pm 0.098$ & $1.83 \pm 0.105$ & $1.86 \pm 0.103$ \\
\hline & AST (IU/L) & $126.1 \pm 30.47$ & $109.5 \pm 15.01$ & $106.0 \pm 19.54$ & $107.2 \pm 16.12$ \\
\hline & ALT (IU/L) & $35.0 \pm 9.63$ & $29.4 \pm 5.17$ & $28.6 \pm 4.56$ & $30.8 \pm 5.60$ \\
\hline & TBIL (mg/dL) & $0.126 \pm 0.0392$ & $0.113 \pm 0.0251$ & $0.140 \pm 0.0193$ & $0.142 \pm 0.0147$ \\
\hline & GGT (IU/L) & $1.15 \pm 1.246$ & $0.43 \pm 0.250$ & $0.34 \pm 0.118$ & $0.31 \pm 0.181$ \\
\hline & $\operatorname{ALP}(\mathrm{IU} / \mathrm{L})$ & $268.3 \pm 57.76$ & $298.8 \pm 69.89$ & $258.3 \pm 57.83$ & $293.5 \pm 68.38$ \\
\hline & $\mathrm{TCHO}(\mathrm{mg} / \mathrm{dL})$ & $65.7 \pm 20.54$ & $57.6 \pm 21.09$ & $56.1 \pm 17.60$ & $49.0 \pm 9.33$ \\
\hline & $\mathrm{TG}(\mathrm{mg} / \mathrm{dL})$ & $41.7 \pm 21.23$ & $27.4 \pm 14.37$ & $34.2 \pm 15.89$ & $24.8 \pm 12.73$ \\
\hline & $\mathrm{Ca}(\mathrm{mg} / \mathrm{dL})$ & $10.90 \pm 0.351$ & $10.78 \pm 0.354$ & $10.77 \pm 0.418$ & $10.95 \pm 0.236$ \\
\hline & $\mathrm{IP}(\mathrm{mg} / \mathrm{dL})$ & $8.97 \pm 0.823$ & $9.41 \pm 1.096$ & $9.83 \pm 0.854$ & $10.04 \pm 0.556^{*}$ \\
\hline & $\mathrm{K}(\mathrm{mmol} / \mathrm{L})$ & $7.24 \pm 1.561$ & $8.17 \pm 1.279$ & $9.19 \pm 0.731^{* *}$ & $9.16 \pm 0.819^{* *}$ \\
\hline & CK (IU/L) & $636.7 \pm 205.21$ & $594.3 \pm 132.46$ & $580.4 \pm 224.39$ & $557.6 \pm 204.43$ \\
\hline & PL (mg/dL) & $100.0 \pm 18.74$ & $89.8 \pm 24.06$ & $90.0 \pm 18.80$ & $83.0 \pm 11.18$ \\
\hline & $\mathrm{Na}(\mathrm{mmol} / \mathrm{L})$ & $142.6 \pm 1.71$ & $142.4 \pm 0.97$ & $142.8 \pm 0.63$ & $143.8 \pm 0.63$ \\
\hline & $\mathrm{Cl}(\mathrm{mmol} / \mathrm{L})$ & $98.7 \pm 1.70$ & $99.1 \pm 1.66$ & $99.6 \pm 1.35$ & $99.8 \pm 1.23$ \\
\hline \multirow{20}{*}{ Female } & GUN (mg/dL) & $124.3 \pm 28.13$ & $122.5 \pm 36.14$ & $120.2 \pm 17.63$ & $123.1 \pm 30.06$ \\
\hline & BUN (mg/dL) & $20.1 \pm 3.43$ & $16.9 \pm 2.02$ & $17.7 \pm 1.54$ & $18.6 \pm 3.32$ \\
\hline & CREA (mg/dL) & $0.59 \pm 0.049$ & $0.52 \pm 0.050 *$ & $0.52 \pm 0.037^{* *}$ & $0.55 \pm 0.060$ \\
\hline & $\mathrm{TP}(\mathrm{g} / \mathrm{dL})$ & $7.38 \pm 0.522$ & $7.35 \pm 0.386$ & $7.48 \pm 0.531$ & $7.38 \pm 0.284$ \\
\hline & $\operatorname{ALB}(\mathrm{g} / \mathrm{dL})$ & $4.72 \pm 0.324$ & $4.78 \pm 0.188$ & $4.91 \pm 0.368$ & $4.80 \pm 0.209$ \\
\hline & A/G (ratio) & $1.77 \pm 0.067$ & $1.86 \pm 0.117$ & $1.91 \pm 0.101$ ** & $1.87 \pm 0.081$ \\
\hline & AST (IU/L) & $115.2 \pm 36.10$ & $122.3 \pm 26.10$ & $98.3 \pm 12.96$ & $105.5 \pm 10.66$ \\
\hline & ALT (IU/L) & $38.9 \pm 29.84$ & $37.8 \pm 18.01$ & $29.6 \pm 8.36$ & $26.8 \pm 4.48$ \\
\hline & TBIL (mg/dL) & $0.133 \pm 0.0215$ & $0.160 \pm 0.0204$ & $0.182 \pm 0.0213 * *$ & $0.194 \pm 0.0399 * *$ \\
\hline & GGT (IU/L) & $0.71 \pm 0.337$ & $0.93 \pm 0.645$ & $0.82 \pm 0.364$ & $0.87 \pm 0.315$ \\
\hline & $\operatorname{ALP}(\mathrm{IU} / \mathrm{L})$ & $170.3 \pm 62.04$ & $137.4 \pm 45.26$ & $169.1 \pm 40.69$ & $174.3 \pm 50.54$ \\
\hline & $\mathrm{TCHO}(\mathrm{mg} / \mathrm{dL})$ & $66.3 \pm 19.06$ & $60.4 \pm 15.15$ & $64.8 \pm 12.15$ & $63.0 \pm 14.01$ \\
\hline & $\mathrm{TG}(\mathrm{mg} / \mathrm{dL})$ & $23.5 \pm 12.20$ & $18.6 \pm 6.68$ & $18.1 \pm 4.18$ & $15.1 \pm 6.62$ \\
\hline & $\mathrm{Ca}(\mathrm{mg} / \mathrm{dL})$ & $11.40 \pm 0.444$ & $11.47 \pm 0.558$ & $11.53 \pm 0.878$ & $11.39 \pm 0.306$ \\
\hline & $\mathrm{IP}(\mathrm{mg} / \mathrm{dL})$ & $7.85 \pm 0.903$ & $8.10 \pm 1.212$ & $8.42 \pm 1.040$ & $7.93 \pm 0.993$ \\
\hline & $\mathrm{K}(\mathrm{mmol} / \mathrm{L})$ & $6.90 \pm 0.611$ & $7.25 \pm 0.799$ & $7.47 \pm 0.913$ & $7.17 \pm 0.691$ \\
\hline & CK (IU/L) & $411.6 \pm 79.44$ & $527.4 \pm 195.99$ & $403.7 \pm 102.16$ & $526.1 \pm 159.18$ \\
\hline & $\mathrm{PL}(\mathrm{mg} / \mathrm{dL})$ & $134.9 \pm 30.32$ & $120.3 \pm 20.72$ & $132.5 \pm 16.77$ & $126.5 \pm 15.52$ \\
\hline & $\mathrm{Na}(\mathrm{mmol} / \mathrm{L})$ & $143.4 \pm 1.35$ & $144.1 \pm 1.66$ & $144.9 \pm 2.02$ & $145.1 \pm 2.18$ \\
\hline & $\mathrm{Cl}(\mathrm{mmol} / \mathrm{L})$ & $101.4 \pm 2.07$ & $102.2 \pm 1.32$ & $101.8 \pm 2.04$ & $102.5 \pm 1.65$ \\
\hline
\end{tabular}

Data are expressed as the mean $\pm \mathrm{SD}, \mathrm{n}=10$ animals per group. ${ }^{*} p<0.05,{ }^{* *} p<0.01$ according to Dunnett's LSD test.

\subsubsection{Gross Autopsy and Histopathological Examinations}

No abnormalities associated with the administration of Yeosinsan were observed by gross autopsy. Regarding visual observation and ophthalmology, no changes associated with the administration of the test substance were observed in any dose group (Table S5).

Under microscopic observations, changes associated with the test substance were observed in the stomach and spleen. In the stomach observation, in the $2000 \mathrm{mg} \cdot \mathrm{kg}^{-1} \cdot \mathrm{d}^{-1}$ 
dose group (G4), squamous cell hyperplasia of the forestomach was observed to be very weak (minimal) to weak (slight). These changes were observed with vesiculation or neutrophil infiltration at the limiting ridge of the forestomach and the glandular ventriculus. In the spleen observation, in the female $500 \mathrm{mg} \cdot \mathrm{kg}^{-1} \cdot \mathrm{d}^{-1}$ group and all dose groups at 1000 and $2000 \mathrm{mg} \cdot \mathrm{kg}^{-1} \cdot \mathrm{d}^{-1}$, extramedullary hematopoiesis was observed to be very weak (minimal) to weak (slight). In the dose groups at or over $500 \mathrm{mg} \cdot \mathrm{kg}^{-1} \cdot \mathrm{d}^{-1}$, increased pigmentation was observed to be very weak (minimal) to weak (slight). In addition, in the male control group and the male $500 \mathrm{mg} \cdot \mathrm{kg}^{-1} \cdot \mathrm{d}^{-1}$ dose group, extramedullary hematopoiesis was observed to be very weak (minimal) in 1 and 10 cases, respectively. This was judged to be a change within the normal range. In the observation of the female genitalia (ovary, uterus, and vagina), changes associated with the test substance were not observed. Other observed findings were judged to be spontaneous or accidental changes observed in animals of similar age, given the incidence (Table 7). Regarding the photomicrographs shown in Figure 4, the chronic high-dose group showed extramedullary hematopoiesis and increased pigmentation in the spleen. The chronic high-dose group showed squamous cell hyperplasia in the forestomach (Figure $4 \mathrm{~L}, \mathrm{R}$ ).

Table 7. Summary of microscopic findings of chronic oral toxicity.

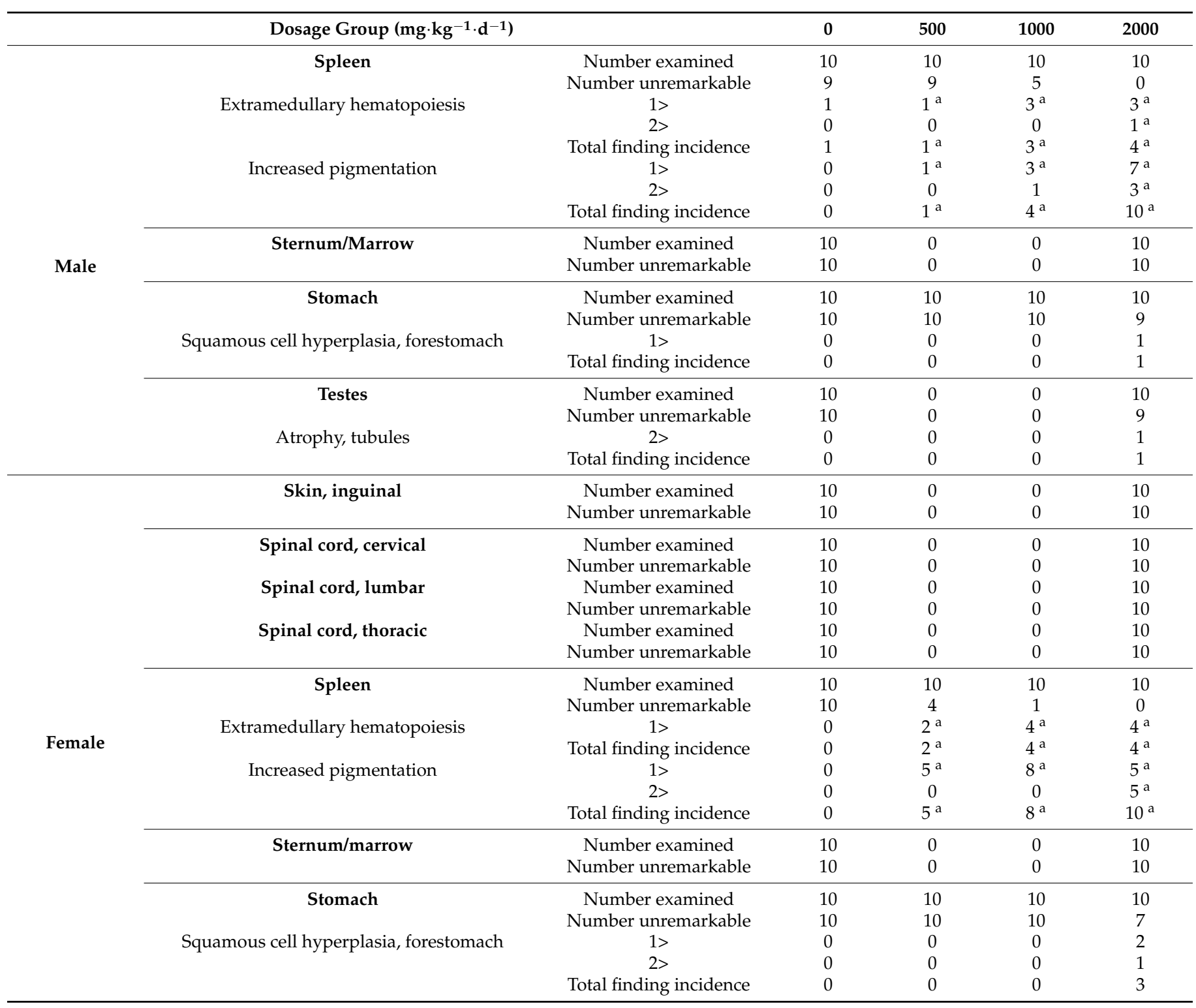


Table 7. Cont

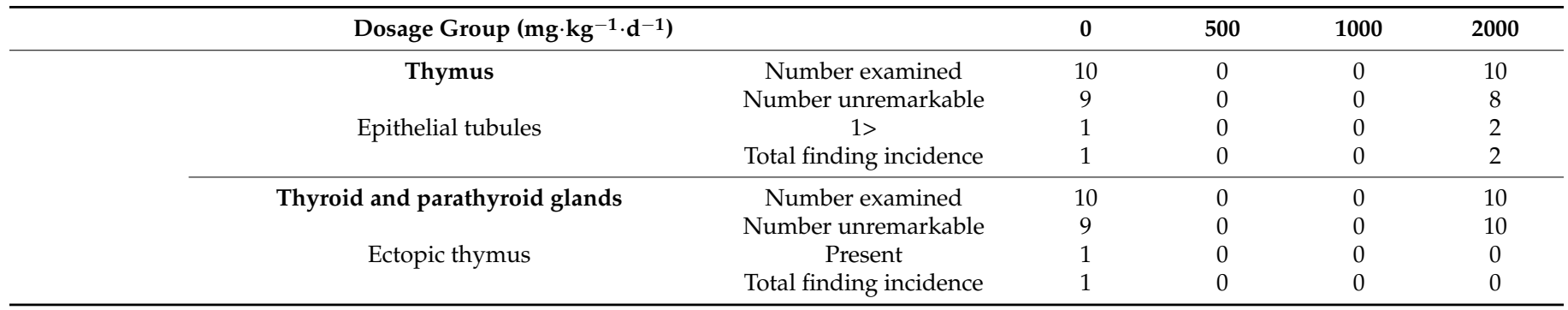

${ }^{a}$ includes the case with multiple symptoms.
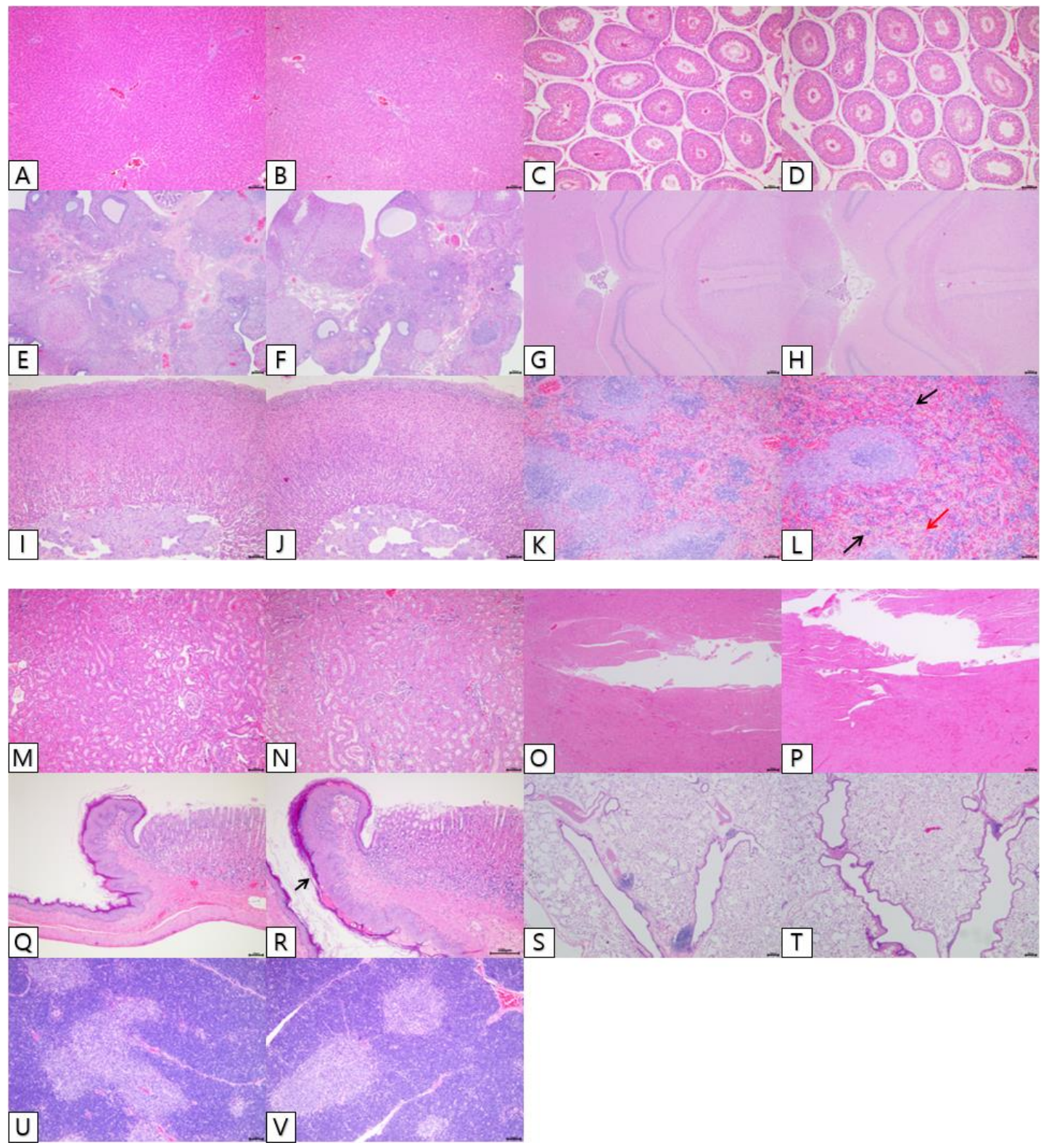

Figure 4. Effects of chronic oral administration of Yeosinsan extract on histopathological evaluation for 91 days. Photomicrographs of representative rats from respective groups: control and $2000 \mathrm{mg} \cdot \mathrm{kg}^{-1} \cdot \mathrm{d}^{-1}$. Liver (A,B), testes (C,D), ovaries $(\mathbf{E}, \mathbf{F})$, brain $(\mathbf{G}, \mathbf{H})$, adrenals $(\mathbf{I}, \mathbf{J})$, spleen $(\mathbf{K}, \mathbf{L})$, kidney $(\mathbf{M}, \mathbf{N})$, heart $(\mathbf{O}, \mathbf{P})$, stomach $(\mathbf{Q}, \mathbf{R})$, lung $(\mathbf{S}, \mathbf{T})$, and thymus $(\mathbf{U}, \mathbf{V})$. The sectioned tissues were Scheme 100. Scale bar $=100 \mu \mathrm{m}$. Spleen in high-dose group (L): Photomicrograph of extramedullary hematopoiesis (black arrow) and increased pigmentation (red arrow). Stomach in the high-dose group (R): Photomicrograph of squamous cell hyperplasia in the forestomach (black arrow). 


\subsubsection{Urine Analysis}

In the urinalysis, in male mice the BILu was found to be $1+$ to $2+$ in 1,5 , and 8 cases of 10 in groups of dose 500,1000 , and $2000 \mathrm{mg} \cdot \mathrm{kg}^{-1} \cdot \mathrm{d}^{-1}$, respectively. In female mice, the BILu was found to be $1+$ to $2+$ in 2,4 , and 5 cases of 10 , at the same dose as the male groups. Abnormal amounts of BILu in the urine are associated with liver disease. In the $2000 \mathrm{mg} \cdot \mathrm{kg}^{-1} \cdot \mathrm{d}^{-1}$ dose group (G4), UBG was observed at about $1+$ in 2 cases of 10 in both males and females. Other observed changes were judged to be unrelated to the test substance because of a lack of correlation with the dose (Table S6).

\section{Discussion}

In this study, Yeosinsan, a traditional herbal formula composed of P. lactiflora and C. rotundus, was evaluated for acute and chronic oral toxicity using female and male SD rats. In the acute toxicity experiment, the main findings (single administration of 0,500 , 1000 , or $2000 \mathrm{mg} / \mathrm{kg}$ of Yeosinsan) indicated no significant alteration in mortality, body weight, general clinical symptoms, or gross pathological signs in either male or female rats. Thus, these results suggest that an approximate acute lethal dose of Yeosinsan is over $2000 \mathrm{mg} / \mathrm{kg}$ in rats.

In the chronic toxicity experiment, the main findings indicated no significant alteration in mortality, body weight, general clinical symptoms, food uptake, ophthalmological condition, organ weight, or gross pathological signs following daily treatment with Yeosinsan $\left(0,500,1000\right.$, or $2000 \mathrm{mg} \cdot \mathrm{kg}^{-1} \cdot \mathrm{d}^{-1}$ for 13 weeks). However, the hematological, clinical, pathological, and histopathological observations demonstrated several significant changes in these groups. In the histopathological experiments, a minimal to slight squamous hyperplasia of the forestomach was found in the $2000 \mathrm{mg} \cdot \mathrm{kg}^{-1} \cdot \mathrm{d}^{-1}$ groups. This observation was accompanied by vesiculation or neutrophil infiltration in the limiting ridge of the forestomach. Thus, the change was considered an adverse effect of inflammatory irritation stimulated by the experimental material, according to guidelines and a previous study $[23,24]$.

Previous studies on the toxicity and safety of P. lactiflora and C. rotundus, the component herbs of Yeosinsan, did not report any severe toxicity [14,22]. A network pharmacology study revealed that $P$. lactiflora has a protective effect against ulcerative colitis [25]. In addition, the combination of P. lactiflora and Inula britannica L. has a protective effect against gastric ulcers induced via the administration of $\mathrm{HCl} / \mathrm{EtOH}$ solution [26]. An herbal formula composed of C. rotundus, Holarrhena antidysenterica L., and Zingiber officinale Rosc. has been used for abdominal pain [14]. However, no previous study reported the protective or deteriorating effect of $C$. rotundus on the stomach. The concentration of $2000 \mathrm{mg} \cdot \mathrm{kg}^{-1} \cdot \mathrm{d}^{-1}$ of Yeosinsan is far from the clinical dose of the ingredient herbs, P. lactiflora and C. rodun$d u s[14,22,27]$. Thus, in general clinical use, Yeosinsan could be regarded as a safe herbal formula up to its usual dose.

In the histopathological observation of the spleen, mild extramedullary hematopoiesis was found in the female $500 \mathrm{mg} \cdot \mathrm{kg}^{-1} \cdot \mathrm{d}^{-1}$ group and all groups at or over $1000 \mathrm{mg} \cdot \mathrm{kg}^{-1} \cdot \mathrm{d}^{-1}$. The change was characterized as the increase in erythroid lineage cells in the red pulp. These findings were considered to be a regenerative reaction to the decrease in the RBC and increases in RETA, RET\%, MCV, and $\mathrm{MCH}$. In addition, very weak increases in pigmentation were observed in the groups at or over $500 \mathrm{mg} \cdot \mathrm{kg}^{-1} \cdot \mathrm{d}^{-1}$. This appears to be related to the clinicopathological findings such as an increase in TBIL in the serum, increases in BILu and UBG in the urine, and an increase in the relative weight of the spleen. These results appear to be related to hemolytic anemia because damaged RBCs are generally removed by macrophages in the spleen [28]. The effects of P. lactiflora or C. rotundus on hemolytic anemia and histopathological changes in the spleen were not previously reported. P. lactiflora has been regarded as a remedy for anemia caused by iron deficiency and hemolysis $[29,30]$. Paeoniflorin and albiflorin, the major compounds of $P$. lactiflora, exhibited a hematopoietic effect on a chemotherapy- or radiotherapy-induced murine myelosuppression model [31,32]. The pathological changes in the spleen induced 
by chemical hemolysis were also reduced by treatment with P. lactiflora or its component glucosides $[30,33]$. C. rotundus also showed anti-hemolytic and hemagglutination-inhibitory effects in a carbapenem-resistant bacteria model [34]. In addition, different extracts of $C$. rotundus did not show any acute toxicity in mice up to a dose of $1 \mathrm{~g} / \mathrm{kg}$ [35].

However, paeoniflorin, a major component of P. lactiflora, has been reported as an anti-thrombotic agent that acts by upregulating urokinase-type plasminogen activator (uPA) [36]. In the theory of traditional Korean and Chinese herbal medicine, the roots of $P$. lactiflora and C. rotundus belong to a group of herbs for removing blood stasis [37,38]. These herbal medicines have been generally used for coagulopathy, and they act by activating anti-coagulating factors including uPA and thereby have side effects such as unexpected bleeding $[37,39]$. Thus, it is possible that a high dose of paeoniflorin, which is contained in Yeosinsan, might be a cause of hemolysis and spleen damage. However, in this study, the RBC counts were slightly decreased and not accompanied by spleen tissue damage. In addition, the findings in the spleen were evaluated as reversible changes owing to adaptive or secondary reactions to hemolysis. Thus, it is believed that the results were not related to the adverse effects of the test material. However, in this study, there are two major limitations. First, the mechanisms underlying the side effects of Yeosinsan were not clearly examined. The issue should be examined by extensive study. Second, Yeosinsan is likely to be used during the initial pregnancy period in clinical practice. Thus, to avoid another toxic side effect on the embryo, additional safety examinations on reproductive toxicity and genotoxicity might be needed for conducting further clinical experiments.

\section{Conclusions}

Based on these data from acute and chronic toxicology studies, the no observed adverse effect level (NOAEL) of the test material, Yeosinsan, was estimated to be $1000 \mathrm{mg} \cdot \mathrm{kg}^{-1} \cdot \mathrm{d}^{-1}$ in both male and female rats. In the previous in vivo efficacy study, 15.96 and $31.92 \mathrm{mg} \cdot \mathrm{kg}^{-1} \cdot \mathrm{d}^{-1}$ of Yeosinsan showed maximal effects on endometrial receptivity and ovarian function, respectively [21]. Because the doses for in vivo efficacy are within the NOAEL in this study, we estimate that Yeosinsan might be safe to use for treating female infertility.

Supplementary Materials: The following are available online at https: / www.mdpi.com/article / 10.3390/app11199325/s1, Table S1: Summary of macroscopic findings of acute oral toxicity, Table S2: The numbers of mortality of acute oral toxicity, Table S3: The numbers of mortality of chronic oral toxicity, Table S4: Effects of chronic oral administration of Yeosinsan extract in relative organ weight (percent of body weight), Table S5: Summary of ophthalmology of chronic oral toxicity, Table S6: Effects of chronic oral administration of Yeosinsan extract in urinalysis.

Author Contributions: M.-J.Y. and B.-K.K. performed the animal toxicological studies. H.J.Y. prepared the materials for the toxicological studies. J.-M.K., J.E.Y. and J.-K.P. analyzed the data and performed the statistical analysis. J.J., B.S.J., and J.-D.H. assisted with technical advice and clinical interpretation. J.-M.K. and K.-T.H. prepared the manuscript. J.-D.H. and K.-T.H. designed and supervised the study. All authors have read and agreed to the published version of the manuscript.

Funding: This work was supported by a 2-Year Research Grant of Pusan National University, and by a grant of the Korea Health Technology R\&D Project through the Korea Health Industry Development Institute (KHIDI), funded by the Ministry of Health and Welfare, Republic of Korea (grant no. HI17C0935).

Institutional Review Board Statement: All animal procedures were approved by the Animal Research Ethics Committee of the Korea Institute of Toxicology (KIT experiment number G217055 and G218038).

Informed Consent Statement: Not applicable.

Data Availability Statement: The data are available upon request to the corresponding author.

Conflicts of Interest: The study was conducted independent of the financial benefit of Cho-A Pharmaceutical Co., the company that supplied the Yeosinsan extract. The funders had no role in 
the design of the study; in the collection, analyses, or interpretation of data; in the writing of the manuscript, or in the decision to publish the results.

\section{References}

1. De Ziegler, D.; Streuli, I.; Meldrum, D.R.; Chapron, C. The value of growth hormone supplements in ART for poor ovarian responders. Fertil. Steril. 2011, 96, 1069-1076. [CrossRef] [PubMed]

2. Coughlan, C.; Ledger, W.; Wang, Q.; Liu, F.; Demirol, A.; Gurgan, T.; Cutting, R.; Ong, K.; Sallam, H.; Li, T.C. Recurrent implantation failure: Definition and management. Reprod. Biomed. Online 2014, 28, 14-38. [CrossRef] [PubMed]

3. Thurston, L.; Abbara, A.; Dhillo, W.S. Investigation and management of subfertility. J. Clin. Pathol. 2019, 72, 579-587. [CrossRef] [PubMed]

4. Xia, J.F.; Inagaki, Y.; Zhang, J.F.; Wang, L.; Song, P.P. Chinese medicine as complementary therapy for female infertility. Chin. J. Integr. Med. 2017, 23, 245-252. [CrossRef]

5. Jeon, S.; Liu, Q.F.; Cai, H.; Jeong, H.J.; Kim, S.H.; Kim, D.I.; Lee, J.H. Administration of a herbal formulation enhanced blastocyst implantation via IkappaB activation in mouse endometrium. Chin. Med. 2020, 15, 112. [CrossRef] [PubMed]

6. Yu, N.; Yang, J.; Yin, T. Extracts from a traditional Chinese herbal remedy (Zhuyun recipe) improve endometrial receptivity in mice with embryonic implantation dysfunction and ovulation stimulation. J. Ethnopharmacol. 2011, 137, 389-395. [CrossRef]

7. Yuan, L.; Feng, F.; Mao, Z.; Huang, J.; Liu, Y.; Li, Y.; Jiang, R. Effects of Erbuzhuyu Decoction Combined with Acupuncture on Endometrial Receptivity Are Associated with the Expression of miR-494-3p. Evid. Based Complement. Altern. Med. 2020, 2020, 9739672. [CrossRef]

8. Huang, L.; Wang, L.; Bao, H.; Xu, Y.; Meng, M.; Qiao, M.; Wang, H.; Kong, S. Traditional Chinese medicine Dingkun Pill facilitates uterine receptivity for implantation in micedagger. Biol. Reprod. 2019, 101, 695-703. [CrossRef]

9. Xin, M.; He, J.; Yang, W.; Yin, X.; Wang, J. Wenshen Yangxue decoction improves endometrial receptivity recovery and promotes endometrial angiogenesis in a rat model. Pharm. Biol. 2018, 56, 573-579. [CrossRef] [PubMed]

10. Lin, J.; Li, X.L.; Song, H.; Li, Q.; Wang, M.Y.; Qiu, X.M.; Li, D.J.; Wang, L. A general description for Chinese medicine in treating premature ovarian failure. Chin. J. Integr. Med. 2017, 23, 91-97. [CrossRef] [PubMed]

11. Zeyneloglu, H.B.; Onalan, G. Remedies for recurrent implantation failure. Semin. Reprod. Med. 2014, 32, 297-305. [CrossRef]

12. Adki, K.M.; Kulkarni, Y.A. Chemistry, pharmacokinetics, pharmacology and recent novel drug delivery systems of paeonol. Life Sci. 2020, 250, 117544. [CrossRef] [PubMed]

13. Xin, Q.; Yuan, R.; Shi, W.; Zhu, Z.; Wang, Y.; Cong, W. A review for the anti-inflammatory effects of paeoniflorin in inflammatory disorders. Life Sci. 2019, 237, 116925. [CrossRef] [PubMed]

14. Pirzada, A.M.; Ali, H.H.; Naeem, M.; Latif, M.; Bukhari, A.H.; Tanveer, A. Cyperus rotundus L.: Traditional uses, phytochemistry, and pharmacological activities. J. Ethnopharmacol. 2015, 174, 540-560. [CrossRef]

15. Seo, W.G.; Pae, H.O.; Oh, G.S.; Chai, K.Y.; Kwon, T.O.; Yun, Y.G.; Kim, N.Y.; Chung, H.T. Inhibitory effects of methanol extract of Cyperus rotundus rhizomes on nitric oxide and superoxide productions by murine macrophage cell line, RAW 264.7 cells. J. Ethnopharmacol. 2001, 76, 59-64. [CrossRef]

16. Choi, H.J.; Chung, T.W.; Park, M.J.; Lee, K.S.; Yoon, Y.; Kim, H.S.; Lee, J.H.; Kwon, S.M.; Lee, S.O.; Kim, K.J.; et al. Paeonia lactiflora Enhances the Adhesion of Trophoblast to the Endometrium via Induction of Leukemia Inhibitory Factor Expression. PLoS ONE 2016, 11, e0148232. [CrossRef]

17. Choi, H.J.; Chung, T.W.; Park, M.J.; Jung, Y.S.; Lee, S.O.; Kim, K.J.; Ha, K.T. Water-extracted tubers of Cyperus rotundus L. enhance endometrial receptivity through leukemia inhibitory factor-mediated expression of integrin alphaVbeta3 and alphaVbeta5. J. Ethnopharmacol. 2017, 208, 16-23. [CrossRef]

18. Arentz, S.; Abbott, J.A.; Smith, C.A.; Bensoussan, A. Herbal medicine for the management of polycystic ovary syndrome (PCOS) and associated oligo/amenorrhoea and hyperandrogenism; a review of the laboratory evidence for effects with corroborative clinical findings. BMC Complement. Altern. Med. 2014, 14, 511. [CrossRef]

19. Arentz, S.; Smith, C.A.; Abbott, J.; Fahey, P.; Cheema, B.S.; Bensoussan, A. Combined Lifestyle and Herbal Medicine in Overweight Women with Polycystic Ovary Syndrome (PCOS): A Randomized Controlled Trial. Phytother. Res. 2017, 31, 1330-1340. [CrossRef]

20. Liao, W.T.; Su, C.C.; Lee, M.T.; Li, C.J.; Lin, C.L.; Chiang, J.H.; Yen, H.R. Integrative Chinese herbal medicine therapy reduced the risk of type 2 diabetes mellitus in patients with polycystic ovary syndrome: A nationwide matched cohort study. J. Ethnopharmacol. 2019, 243, 112091. [CrossRef]

21. Hee Jung, C.; Bo Sun, J.; Mi Ju, P.; Min Jung, P.; Boram, B.; Bo Sung, K.; Hye Rin, P.; Keuk Jun, K.; Hee Jin, Y.; Jeong Eun, Y.; et al. Yeosin-san Increases Female Fertility through Inducing Uterine Receptivity and Ovarian Function. J. Physiol. Pathol. Korean Med. 2019, 33, 141-150. [CrossRef]

22. Parker, S.; May, B.; Zhang, C.; Zhang, A.L.; Lu, C.; Xue, C.C. A Pharmacological Review of Bioactive Constituents of Paeonia lactiflora Pallas and Paeonia veitchii Lynch. Phytother. Res. 2016, 30, 1445-1473. [CrossRef]

23. Boorman, G.A.; Hong, H.L.; Jameson, C.W.; Yoshitomi, K.; Maronpot, R.R. Regression of methyl bromide-induced forestomach lesions in the rat. Toxicol. Appl. Pharm. 1986, 86, 131-139. [CrossRef]

24. McConnell, E.E.; Solleveld, H.A.; Swenberg, J.A.; Boorman, G.A. Guidelines for combining neoplasms for evaluation of rodent carcinogenesis studies. J. Natl. Cancer Inst. 1986, 76, 283-289. [PubMed] 
25. Zhang, Y.; Li, X.; Xu, X.; Yang, N. Mechanisms of Paeonia lactiflora in Treatment of Ulcerative Colitis: A Network Pharmacological Study. Med. Sci. Monit. 2019, 25, 7574-7580. [CrossRef] [PubMed]

26. Kim, Y.S.; Park, H.J.; Kim, H.; Song, J.; Lee, D. Gastroprotective Effects of Paeonia Extract Mixture HT074 against Experimental Gastric Ulcers in Rats. Evid. Based Complement. Altern. Med. 2019, 2019, 3546258. [CrossRef]

27. Kamala, A.; Middha, S.K.; Karigar, C.S. Plants in traditional medicine with special reference to Cyperus rotundus L.: A review. 3Biotech 2018, 8, 309. [CrossRef]

28. Everds, N.E. Evaluation of clinical pathology data: Correlating changes with other study data. Toxicol. Pathol. 2015, 43, 90-97. [CrossRef]

29. Ye, X.W.; Deng, Y.L.; Xia, L.T.; Ren, H.M.; Zhang, J.L. Uncovering the mechanism of the effects of Paeoniae Radix Alba on iron-deficiency anaemia through a network pharmacology-based strategy. BMC Complement. Med. 2020, 20, 130. [CrossRef]

30. Ku, S.K.; Kim, H.; Kim, J.W.; Kang, K.S.; Lee, H.J. Ameliorating effects of herbal formula hemomine on experimental subacute hemorrhagic anemia in rats. J. Ethnopharmacol. 2017, 198, 205-213. [CrossRef] [PubMed]

31. Zhu, Y.; Wang, L.; Yang, Z.; Wang, J.; Li, W.; Zhou, J.; Zhang, J. Hematopoietic Effects of Paeoniflorin and Albiflorin on Radiotherapy-Induced Myelosuppression Mice. Evid. Based Complement. Altern. Med. 2016, 2016, 5789381. [CrossRef]

32. Zhu, Y.L.; Wang, L.Y.; Wang, J.X.; Wang, C.; Wang, C.L.; Zhao, D.P.; Wang, Z.C.; Zhang, J.J. Protective effects of paeoniflorin and albiflorin on chemotherapy-induced myelosuppression in mice. Chin. J. Nat. Med. 2016, 14, 599-606. [CrossRef]

33. Kang, L.; Miao, J.X.; Cao, L.H.; Miao, Y.Y.; Miao, M.S.; Liu, H.J.; Xiang, L.L.; Song, Y.G. Total glucosides of herbaceous peony (Paeonia lactiflora Pall.) flower attenuate adenine- and ethambutol-induced hyperuricaemia in rats. J. Ethnopharmacol. 2020, 261, 113054. [CrossRef] [PubMed]

34. Thakur, P.; Chawla, R.; Narula, A.; Goel, R.; Arora, R.; Sharma, R.K. Anti-hemolytic, hemagglutination inhibition and bacterial membrane disruptive properties of selected herbal extracts attenuate virulence of Carbapenem Resistant Escherichia coli. Microb. Pathog. 2016, 95, 133-141. [CrossRef] [PubMed]

35. Soumaya, K.J.; Dhekra, M.; Fadwa, C.; Zied, G.; Ilef, L.; Kamel, G.; Leila, C.G. Pharmacological, antioxidant, genotoxic studies and modulation of rat splenocyte functions by Cyperus rotundus extracts. BMC Complement. Altern. Med. 2013, 13, 28. [CrossRef]

36. Ye, S.; Mao, B.; Yang, L.; Fu, W.; Hou, J. Thrombosis recanalization by paeoniflorin through the upregulation of urokinasetype plasminogen activator via the MAPK signaling pathway. Mol. Med. Rep. 2016, 13, 4593-4598. [CrossRef] [PubMed]

37. Park, B.; You, S.; Jung, J.; Lee, J.A.; Yun, K.J.; Lee, M.S. Korean studies on blood stasis: An overview. Evid. Based Complement. Altern. Med. 2015, 2015, 316872. [CrossRef]

38. Liu, Y.; Yin, H.J.; Shi, D.Z.; Chen, K.J. Chinese herb and formulas for promoting blood circulation and removing blood stasis and antiplatelet therapies. Evid. Based Complement. Altern. Med. 2012, 2012, 184503. [CrossRef]

39. Jiang, Y.; Zhang, G.; Yan, D.; Yang, H.; Ye, Z.; Ma, T. Bioactivity-Guided Fractionation of the Traditional Chinese Medicine Resina Draconis Reveals Loureirin B as a PAI-1 Inhibitor. Evid. Based Complement. Altern. Med. 2017, 2017, 9425963. [CrossRef] 